\title{
Hadronic uncertainties in semileptonic $B$ decays
}

\author{
Jorge Martin Camalich* \\ PRISMA Cluster of Excellence \& Institut für Kernphysik, \\ Johannes Gutenberg Universität Mainz, 55128 Mainz, Germany \\ E-mail: camalich@uni-mainz.de
}

\begin{abstract}
Anomalies in (semi)leptonic $B$-meson decays present interesting patterns that might be revealing the shape of the new physics to come. I will review the decays and observables where these appear, discussing the extent up to which the respective standard model predictions are understood, especially regarding the contributions from hadronic matrix elements. In this sense, the most interesting anomalies are the violation of lepton universality suggested by a recent measurement of a deficit of $B^{+} \rightarrow K^{+} \mu \mu$ over $B^{+} \rightarrow K^{+} e e$ decays or of surplus of $B \rightarrow D^{(*)} \tau \nu$ over $B \rightarrow D^{(*)} \ell v$. This raises very interesting questions concerning the lepton-flavor structure of the presumed new interactions, some of which I will address in the context of effective operators. Finally, I will discuss new possible experiments that could unambiguously confirm and characterize the putative new-physics effect.
\end{abstract}

16th International Conference on B-Physics at Frontier Machines

2-6 May 2016

Marseille, France

${ }^{*}$ Speaker. 


\section{Introduction}

The last years have witnessed a very rapid progress, both in experiment and theory, of the interpretation and search of new physics (NP) in (semi) leptonic $B$-decays. While in the highenergy frontier we are lacking from very exciting news, in $B$-physics there are some tensions with the predictions of the standard model (SM). The first type of anomalies appear in the rare $b \rightarrow$ sll transitions, in the angular analysis of the $B \rightarrow K^{*} \mu \mu$ decay [1] and as deficits in the $B \rightarrow K \mu \mu$ and $B_{s} \rightarrow \phi \mu \mu$ branching fractions and in the ratio [2]:

$$
R_{K}=\frac{\mathscr{B}\left(B^{+} \rightarrow K^{+} \mu \mu\right)}{\mathscr{B}\left(B^{+} \rightarrow K^{+} e e\right)} .
$$

These measurements have been claimed to be in $\sim 4 \sigma$ tension with the SM [3]. This would naively correspond to the tree-level exchange of an $\sim \mathscr{O}(10 \mathrm{TeV})$ neutral particle selectively coupled to muons.

The second type of anomalies appear in the charged-current $b \rightarrow c \tau \nu$ transitions, in the ratios

$$
R_{D^{(*)}}=\frac{\mathscr{B}\left(\bar{B} \rightarrow D^{(*)} \tau^{-} \bar{v}\right)}{\mathscr{B}\left(\bar{B} \rightarrow D^{(*)} \ell^{-} \bar{v}\right)}, \text { with } \ell=e, \mu,
$$

which have been measured to be enhanced with respect to the SM in the two channels ( $B D$ and $\left.B D^{*}\right)$ by BaBar [4] and Belle [5] and in the $B D^{*}$ one by LHCb [6]. The average of the measurements currently stands at $\sim 4 \sigma$ and it would naively correspond to the tree-level exchange of an $\sim \mathscr{O}(\mathrm{TeV})$ new charged particle selectively coupled to $\tau$ leptons.

Thus, an intriguing feature of these anomalies is that they appear in lepton universality ratios. This, in general, is expected to lead to other signals of flavor violation which have not been yet reported e.g. in very precise universality tests performed with pion, kaon and weak boson decays [7]. This suggests that the putative model beyond the SM should have a non-trivial flavor structure, e.g. $[8,9,10,11,12,13,14,15,16,17]$ and references therein.

When analyzing the $B$-decays we are not just probing the short-distance structure of the weak $b$-quark decay but also testing our understanding of the more prosaic long-distance strongdynamics governing the hadron in which it occurs. In this sense, the lattice QCD (LQCD) community is making very rapid progress in the calculation of many of the relevant hadronic matrix elements appearing in $B$ - and other weak hadron decays [18]. In addition, effective field theories (EFT) simplify the calculation of these quantities and provide accurate and systematic approximations to full QCD in some kinematic limit. Beyond LQCD and EFTs, one needs to rely on nonperturbative approaches like light-cone sum rules (LCSR) which incorporate first-principles material to a large extent but that need to introduce model-dependent steps in order to extract a given hadronic quantity. In the following, I will give a brief overview on the current status of the role of the hadronic uncertainties in relevant semileptonic $B$ decays.

2. $B_{s} \rightarrow \mu \mu$

The simplest $b \rightarrow s \ell \ell$ transition one can study is the leptonic rare decay $B_{s} \rightarrow \mu \mu$ :

$$
\mathscr{B}_{s l} \simeq \frac{G_{F}^{2} \alpha^{2}}{64 \pi^{3}} \tau_{B_{s}} m_{B_{s}}^{3} f_{B_{s}}^{2}\left|V_{t b} V_{t s}^{*}\right|^{2}\left[\left|C_{S}-C_{S}^{\prime}\right|^{2}+\left|C_{S}+C_{S}^{\prime}-\frac{2 m_{l}}{m_{B_{s}}}\left(C_{10}-C_{10}^{\prime}\right)\right|^{2}\right]
$$


where we have assumed that the NP appear at a energy scale much larger than the EW scale in the construction of the effective operators; namely, we are working in the context of the SMEFT [19, 20]. On top of the loop- and GIM-suppressions, this mode is helicity-suppressed in the SM (through $C_{10}$ ), which makes it to be very sensitive to any NP producing (pseudo)scalar effective contributions $C_{S}^{(\prime)}$. The hadronic matrix that enters in the decay is the $B_{s}$ decay constant $f_{B_{s}}$ which has been calculated accurately by different LQCD collaborations, e.g. $f_{B_{s}}=228.4(3.7) \mathrm{MeV}$ in the latest $N_{f}=2+1$ FLAG average [18]. This, combined with the latest calculation of the EW and QCD corrections to the matching of $C_{10}$ [21], allows for a very accurate prediction of this decay in the $\mathrm{SM}, \overline{\mathscr{B}}_{s \mu}^{\mathrm{SM}}=3.65(23) \times 10^{-9}[21] .^{1}$

Despite its rarity, the $B_{s} \rightarrow \mu \mu$ decay has been experimentally discovered at the LHC, where the CMS and LHCb combined analysis finds $\overline{\mathscr{B}}_{s \mu}^{\text {expt }}=2.9(7) \times 10^{-9}[23,24]$. These groundbreaking measurements are in agreement with the SM prediction above, and this can be translated into bounds on NP up to scales as high as $\sim \mathscr{O}(100) \mathrm{TeV}[20]$.

\section{3. $B \rightarrow K \ell \ell$ and $R_{K}$}

The semileptonic decays are much more challenging to investigate theoretically than their leptonic siblings. Instead of a disintegration of a $B_{q}$ meson into the QCD vacuum, we have the hadronic matrix element describing a flavor transition from a $B$ meson into a light hadron. These are not simply parametrized by constants but by functions of the $q^{2}$ of the 3-body decay or form factors. For the $B \rightarrow K \ell \ell$ decay, $q^{2}=\left(p_{B}-p_{K}\right)^{2} \in\left[4 m_{\ell}^{2},\left(m_{B}-m_{K}\right)^{2}\right]$ spans a large interval of kinematic situations introducing different hierarchies of scales based on which we can perform different approximations of QCD. For instance, in the high- $q^{2}$ region, the $K$ meson recoils softly in the $B$ rest frame and one can apply HQEFT or LQCD, whereas in the low- $q^{2}$ region the kaon recoils very energetically and one can use SCET or QCD factorization, and it is the range of applicability of the LCSR. Furthermore, one also needs to take into account the effects of the "current-current" fourquark operators (dubbed $\mathscr{O}_{1}$ and $\mathscr{O}_{2}$ ) stemming from the $b \rightarrow s c \bar{c}$ transition that is not suppressed by neither mixing angles nor loop factors in the SM. They contribute to $b \rightarrow s \ell \ell$ amplitudes via,

$$
\mathscr{T}_{i}^{\mu}=i \int d^{4} x e^{i q \cdot x} T\left\{\mathscr{O}_{i}(0), j_{\mathrm{em}}^{\mu}(x)\right\}
$$

where the dilepton pair is produced by the off-shell photon from the electromagnetic current. These matrix elements receive dominant contributions from long-distance fluctuations of the charm-quark fields that manifest through charmonium resonances in the intermediate and high- $q^{2}$ regions.

In spite of these difficulties, one can still find an observable in $B \rightarrow K \ell \ell$ that is almost free from hadronic uncertainties. Indeed, as long as one stays away from the low- $q^{2}$ end point where the phase space effects are important, the $R_{K}$ ratio defined in eq. (1.1) is predicted to be 1 in the SM regardless of the hadronic uncertainties which cancel almost exactly in the ratio [25]. This has been measured by LHCb in the $q^{2}$ bin $[1,6] \mathrm{GeV}^{2}$ finding $\left.R_{K}\right|^{\mathrm{LHCb}}=0.745_{-0.074}^{+0.090}$ (stat) \pm 0.036 (syst) [2], which stands in stark contradiction with the SM prediction with a significance of $2.6 \sigma .^{2}$

\footnotetext{
${ }^{1}$ The overline refers to the fact that the experiment access the time-integrated branching ratios which depend on the details of $B_{S} \bar{B}_{S}$ mixing [22].

${ }^{2}$ The EM radiative corrections change the SM prediction at the few-percent level [26].
} 
In the context of the SMEFT, there are no dimension- 6 tensor operators, which otherwise enter in the $B \rightarrow K \ell \ell$ rate; scalar operators cannot produce this effect or it would upset the constraints from the $B_{s} \rightarrow \ell \ell$ decays above. Hence, the putative NP effect must come from a lepton-specific contribution to the semileptonic current operators $\mathscr{O}_{9,10}^{(\prime)}$. For example, a contribution of the type $C_{9}^{\mu} \simeq-1$ implies $R_{K} \simeq 0.75$ [20].

\section{4. $B \rightarrow K^{*} \ell \ell$ and $P_{5}^{\prime}$}

The $B \rightarrow K^{*} \ell \ell$ is also a semileptonic decay and it involves the difficulties with hadronic matrix elements discussed above. However, this is a decay much richer than $B \rightarrow K \ell \ell$ because the $K^{*}$ carries polarization and it decays strongly. The final 4-body differential decay rate contains 12 $q^{2}$-dependent observables $I_{i}\left(q^{2}\right)$, (plus another 12 from the $C P$-conjugate mode), all sensitive to the same Wilson coefficients and the same form factors and "charm" contributions. Namely, the helicity amplitudes of the decay can be written as [27, 28],

$$
\begin{aligned}
& H_{V}(\lambda)=-i N\{\overbrace{\left[C_{C_{9}} \tilde{V}_{L \lambda}+\frac{m_{B}^{2}}{q^{2}} h_{\lambda}\right]}^{C_{9}^{\text {eff }}}+\frac{\hat{m}_{b} m_{B}}{q^{2}} C_{7} \tilde{T}_{L \lambda}\}, \\
& H_{A}(\lambda)=-i N C_{10} \tilde{V}_{L \lambda}, \quad H_{P}=i N \frac{2 m_{l} \hat{m}_{b}}{q^{2}} C_{10}\left(\tilde{S}_{L}+\frac{m_{s}}{m_{b}} \tilde{S}_{R}\right),
\end{aligned}
$$

where $\lambda$ denotes the helicity of the $K^{*}$ and $V, A$ and $P$ indicates whether the coupling of the current to the leptons is vector, axial or pseudoscalar, respectively. There are 7 independent form factors and the charm appears in terms of the helicity projections $h_{\lambda}$ of eq. (3.1). It is important to remember that $\mathscr{O}_{1,2}$ mix into $\mathscr{O}_{9}$ under QCD [29] and the resulting scale dependence in $C_{9}$ must be canceled by the one of $h_{\lambda}[30,31]$. This implies that, individually, none of the two contributions (but their combination $C_{9}^{\text {eff }}$ ) is observable and, in order to extract this Wilson coefficient from the data, one needs an accurate calculation of the "charm".

At low- $q^{2}$ one can calculate both contributions in the strict heavy-quark limit within the framework of QCD factorization [32, 30]. At leading order in $\alpha_{s}$ the form factors can be expressed in terms of only two reduced "soft" form factors, customarily labeled as $\xi_{\perp}$ and $\xi_{\|}$[33]. The $\alpha_{s^{-}}$ corrections are calculable in perturbation theory but an extension of factorization including a systematic treatment of the $\mathscr{O}\left(\Lambda / m_{b}\right)$ power corrections remains unknown [32]. Therefore, we need to estimate them by power counting arguments [28], exploiting all possible relations among the form factors in QCD, or use LCSR [34] (or any other nonperturbative method) to calculate or estimate their size.

There also exists a factorization treatment for the corrections in eq. (3.1) [30]. For the charm, this is valid as long as the $c \bar{c}$ fluctuations produced by $\mathscr{O}_{1,2}$ have a large virtuality, which corresponds to the $q^{2}$ region well below the $c \bar{c}$ threshold and that is almost generally accepted to lie below $q^{2}=6 \mathrm{GeV}^{2}$. Power corrections to QCD factorization, incarnated by the soft-gluon exchange between the charm and the $B \rightarrow K^{*}$ hadronic transition, can also be calculated within the LCSR [35] and have been shown to scale as $\mathscr{O}\left(\Lambda^{2} /\left(4 m_{c}\right)^{2}\right) \sim \mathscr{O}\left(\Lambda / m_{b}\right)$. These results can be 

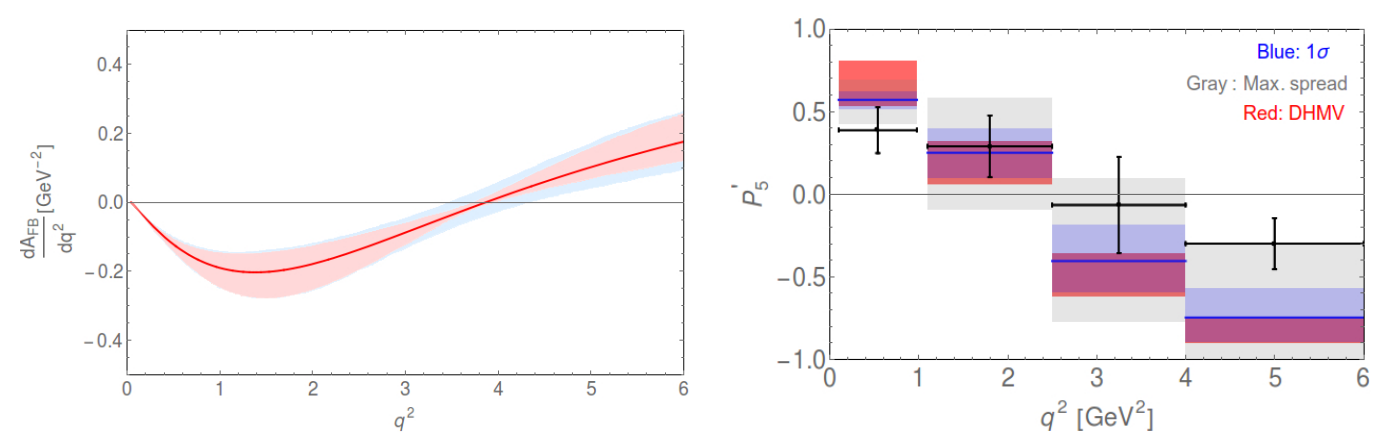

Figure 1: Left panel: Leptonic forward-backward asymmetry in the SM with the theoretical errors at $1 \sigma$ produced by the QCD factorization inputs, especially $\xi_{X}$ (red band), and by power corrections (blue band), and assuming they distribute normally. Right panel: Binned results for $P_{5}^{\prime}$ compared to the LHCb data and using different assumptions for the power corrections.

used to parametrize and estimate the effect of the power corrections to the charm but it has to be emphasized that the LCSR calculation is only valid for $q^{2} \ll 4 m_{c}^{2}[27,28]$.

For the discussion of the phenomenological consequences of the hadronic uncertainties let us start from the strict HQ limit and neglect the $\alpha_{s}$ corrections. Since the 24 observables $I_{i}$ 's can depend on either $\xi_{\perp}^{2}, \xi_{\|}^{2}$ or $\xi_{\perp} \xi_{\|}$, one can engineer ratios for which, in this approximation, soft form factors cancel, so that they exclusively depend on combinations of Wilson coefficients. ${ }^{3}$ These optimized observables conform the $P_{i}^{\left({ }^{\prime}\right)}$-basis [36] and they generalize the same property first discussed for the leptonic forward-backward asymmetry, $A_{F B}$, at the particular $q^{2}$ in which it crosses 0 , and which is distorted by the $\alpha_{s}$ and the power corrections. ${ }^{4}$

Let us introduce $P_{5}^{\prime}$ in the HQ expansion [28]:

$$
\begin{aligned}
& P_{5}^{\prime} \equiv \frac{I_{5}}{2 \sqrt{-I_{2 s} I_{2 c}}}=\left.P_{5}^{\prime}\right|_{\infty}\left(1+\frac{a_{V_{-}}-a_{T_{-}}}{\xi_{\perp}} \frac{m_{B}}{|\vec{k}|} \frac{m_{B}^{2}}{q^{2}} C_{7}^{\mathrm{eff}} \frac{C_{9, \perp} C_{9, \|}-C_{10}^{2}}{\left(C_{9, \perp}^{2}+C_{10}^{2}\right)\left(C_{9, \perp}+C_{9, \|}\right)}\right. \\
& \left.+\frac{a_{V_{0}}-a_{T_{0}}}{\xi_{\|}} 2 C_{7}^{\mathrm{eff}} \frac{C_{9, \perp} C_{9, \|}-C_{10}^{2}}{\left(C_{9, \|}^{2}+C_{10}^{2}\right)\left(C_{9, \perp}+C_{9, \|}\right)}+8 \pi^{2} \frac{\tilde{h}_{-}}{\xi_{\perp}} \frac{m_{B}}{|\vec{k}|} \frac{m_{B}^{2}}{q^{2}} \frac{C_{9, \perp} C_{9, \|}-C_{10}^{2}}{C_{9, \perp}+C_{9, \|}}+\ldots\right)+\mathscr{O}\left(\Lambda^{2} / m_{B}^{2}\right),
\end{aligned}
$$

where $\left.P_{5}^{\prime}\right|_{\infty}, C_{9, \|}$ and $C_{9, \perp}$ are combinations of Wilson coefficients, and the $a_{X}$ are parameters for the power corrections to the form factors $X$. Thus, the bulk of the uncertainty to the optimized observables does not stem from the soft form factors (as the rate or the $I_{i}$ 's) but from the power corrections and the charm [28]. This is illustrated in Fig. 1. On the left panel we plot $d A_{F B} / d q^{2}$ where the hadronic uncertainty is dominated by $\xi_{X}$ (red band) except for the region about the zerocrossing where the main contribution to the error stems from the power corrections (blue band). On the right-hand panel we show the binned results for $P_{5}^{\prime}$, with different assumptions on the theoretical errors and compared to the experimental data [1], which is in tension with the SM predictions labeled as DHMV [37] in the higher $q^{2}$ bin. This tension is enhanced if one decides to use data

\footnotetext{
${ }^{3} \mathrm{We}$ are neglecting here the contribution of the weak-annihilation graph at $\alpha_{s}^{0}$ which is double-suppressed by CKM factors or by small QCD penguins.

${ }^{4}$ It is important to emphasize, though, that some observables, called $P_{1}$ and $P_{3}^{\mathrm{CP}}$, are sensitive to the "wrong-helicity" amplitudes. They receive hadronic corrections to the HQ limit from the leading power corrections which are further suppressed around the low- $q^{2}$ endpoint [27, 28], providing a theoretically clean probe of right-handed NP currents.
} 
above $q^{2}=6 \mathrm{GeV}^{2}$, and leads to the $P_{5}^{\prime}$ anomaly, which can be explained by a NP contribution of the same type as for $R_{K}, C_{9}^{\mu} \simeq-1$. This coincidence is remarkable and also the fact that it remains in global fits to $b \rightarrow s \ell \ell$ data $[3,38]$. However, as shown in the figure, the significance of the tension in $P_{5}^{\prime}$ (and similar observables) crucially depends on assumptions made on the power corrections. Besides reasonable corrections to the form factors [28], uncontrolled contributions from the charm can help to explain the tension $[39,40]$. The latter is especially important due to the difficulty to calculate them model-independently as soon as we approach the $c \bar{c}$ threshold as well as for the observation made above that only the combination $C_{9}^{\text {eff }}$ is truly observable.

\section{5. $B_{s}^{*} \rightarrow \ell \ell$ and backwards}

At high $q^{2}$ one can analytically continue eq. (3.1) into the complex $q^{2}$-plane to perform an operator product expansion (OPE) [31]. The result is then continued back to the real $q^{2}$ and gives the physical rates invoking "quark-hadron duality", whose validity is justified if $q^{2}$ is well above the resonant contributions. Violations to quark-hadron duality are difficult to estimate and important for the semileptonic $b \rightarrow$ sll decays, for which $\sqrt{q^{2}} \leq m_{B}-m_{K} \lesssim 4790 \mathrm{MeV}$, close to the $X(4660)$ charmonium resonance [41, 42] (for a recent and more comprehensive phenomenological analysis see [43]).

In ref. [44] a method circumventing this problem and providing a theoretically clean measurement of $C_{9}\left(m_{b}\right)$ was proposed. It is based on measuring the rare decays of the excited $B$ mesons namely, the $B_{s}^{*} \rightarrow \ell \ell$. Theoretically these modes are very clean because ( $i$ ) the amplitude only depends on decay constants obtained from LQCD; and (ii) the invariant mass of the process is well above the charmonium resonances and the application of the OPE for eq. (3.1) is well justified. This allows to predict the decay rate very accurately: $\Gamma_{\ell \ell}=1.12(8) 10^{-18} \mathrm{GeV}$. Experimentally, measuring the decay rate is very challenging as the rare weak decay has to compete with the dominant electromagnetic decay $B_{s}^{*} \rightarrow B_{s} \gamma$ and the resulting branching fraction falls in $\sim 10^{-11}$. Alternatively, one could measure the rate using resonant $\ell^{+} \ell^{-} \rightarrow B_{s}^{*} \rightarrow B_{s} \gamma$ scattering since the strong suppression of the rare processes is compensated by a large enhancement from the small width of the resonance. The estimated (effective) cross-sections could be of the order of $1-10$ ab for the current or projected accelerators [44].

\section{6. $B \rightarrow D^{(*)} \tau \nu$ and $R_{D^{(*)}}$}

We end our discussion of hadronic uncertainties with the $b \rightarrow c \tau v$ transitions and the decays $B \rightarrow D^{(*)} \tau \nu$ measured through the ratios $R_{D^{(*)}}$ in eq. (1.2). These are lepton-universality ratios, analogous to $R_{K}$, but with the caveat that the $\tau$-mass effects are not negligible in any kinematic region of the decay. An additional important simplification is that this is a charged-current transition and one does not have to deal with QCD contributions of the type in eq. (3.1). The standard procedure to treat these decays is to fit model-independent parametrizations of the form factors using the light-lepton modes [45, 46]. One then plugs the results into the $R_{D^{(*)}}$ ratios, in which the uncertainties largely cancel. The main theoretical uncertainty in the SM stems from scalar form factors, that for $J_{V-A}^{\mu}$, go like $\sim f_{0}\left(q^{2}\right) q^{\mu}$ (for the $B D$ mode). The $q^{\mu}$ becomes $m_{\ell}$ when it hits the leptonic matrix element (equation of motion), and it is negligible for muons and electrons but not 
for the $\tau$. Luckily, LQCD have made important progress in the calculation of these form factors in the low-recoil limit, while at $q^{2}$ the scalar (pseudoscalar) form factors can be connected to the other ones using CVC (PCAC) [47, 48, 49]. These two facts combined reduce the uncertainties and, in fact, it is very difficult to interpret the $R_{D^{(*)}}$ anomalies as uncontrolled hadronic effects.

On the other hand, these modes are experimentally quite difficult as the $\tau$ must be reconstructed from the decay products. This can be tackled from a theoretical perspective. For instance the full measurable kinematic distributions of the final 5-body $\bar{B} \rightarrow D^{(*)} \tau^{-}\left(\rightarrow \ell^{-} \bar{v}_{\ell} v_{\tau}\right) \bar{v}_{\tau}$ decay rate has been recently obtained analytically and a new integrated observable based on the forward-backward asymmetry of the final lepton has been proposed [50].

\section{Conclusions}

We are witnessing a very interesting time in flavor phenomenology as various signals of nonSM physics might be showing up in $B$ decays. The main difficulty with the interpretation of these processes is that they are afflicted by hadronic uncertainties which need to be carefully assessed. Some of the tensions appear in $R_{K}$ and $R_{D^{(*)}}$ which are lepton-universality ratios and for which one can offer clean predictions in the SM. But for other observables, based on the differential rates, the tensions with the data depend significantly on the assumptions made for the hadronic uncertainties. In light of this, one may ask how will we eventually convince ourselves that the tensions are produced by NP. I can see three possible scenarios:

- We find new particles at the LHC that can easily explain the signals in $B$ decays under general assumptions. This is a very interesting scenario as the interplay between collider and flavor data will be crucial to unravel the structure of the NP.

- Another interesting scenario is that NP is confirmed in various lepton-universality ratios or observables as clean as $B_{s} \rightarrow \mu \mu$, even though nothing else is discovered at the LHC. Besides deepening our understanding of the structure and relevance of flavor in nature, we would have an energy scale at which new degrees of freedom should show up.

- The least optimistic scenario by far is that we neither confirm the signal of NP for those clean observables nor we find new particles at the LHC. In this case it could prove difficult to convincingly demonstrate that there is NP in these modes, especially if it shows up in $C_{9}$. New theoretical breakthroughs, for example extending factorization to the $\Lambda / m_{b}$ corrections, or new ideas, like $B_{s}^{*} \rightarrow \ell \ell$, could be necessary to settle the issue.

\section{Acknowledgments}

I would like to thank R. Alonso, B. Grinstein, S. Jäeger and A. Kobach with whom I have collaborated in these topics. I received funding from the People Programme (Marie Curie Actions) of the European Union's Seventh Framework Programme (FP7/2007-2013) under REA grant agreement $n$ PIOF-GA-2012-330458. 


\section{References}

[1] Roel Aaij et al., Angular analysis of the $B^{0} \rightarrow K^{* 0} \mu^{+} \mu^{-}$decay using $3 \mathrm{fb}^{-1}$ of integrated luminosity, JHEP, 02 104, (2016).

[2] Roel Aaij et al., Test of lepton universality using $B^{+} \rightarrow K^{+} \ell^{+} \ell^{-}$decays, Phys.Rev.Lett., 113(15) 151601, (2014).

[3] Sébastien Descotes-Genon, Lars Hofer, Joaquim Matias, and Javier Virto, Global analysis of $b \rightarrow$ sll anomalies, JHEP, 06 092, (2016).

[4] J.P. Lees et al., Measurement of an Excess of $\bar{B} \rightarrow D^{(*)} \tau^{-} \bar{v}_{\tau}$ Decays and Implications for Charged Higgs Bosons, Phys.Rev., D88(7) 072012, (2013).

[5] M. Huschle et al., Measurement of the branching ratio of $\bar{B} \rightarrow D^{(*)} \tau^{-} \bar{v}_{\tau}$ relative to $\bar{B} \rightarrow D^{(*)} \ell^{-} \bar{v}_{\ell}$ decays with hadronic tagging at Belle, Phys. Rev., D92(7) 072014, (2015).

[6] Roel Aaij et al., Measurement of the ratio of branching fractions $\left.\mathscr{B}\left(\bar{B}^{0} \rightarrow D^{*+} \tau^{-} \bar{v}_{\tau}\right)\right) / \mathscr{B}\left(\bar{B}^{0} \rightarrow D^{*+} \mu^{-} \bar{v}_{\mu}\right),,(2015)$.

[7] MartÃ n GonzÃąlez-Alonso and Jorge Martin Camalich, Global Effective-Field-Theory analysis of New-Physics effects in (semi)leptonic kaon decays, , (2016).

[8] Wolfgang Altmannshofer, Stefania Gori, Maxim Pospelov, and Itay Yavin, Quark flavor transitions in $L_{\mu}-L_{\tau}$ models, Phys. Rev., D89 095033, (2014).

[9] Sheldon L. Glashow, Diego Guadagnoli, and Kenneth Lane, Lepton Flavor Violation in B Decays?, Phys. Rev. Lett., 114 091801, (2015).

[10] Bhubanjyoti Bhattacharya, Alakabha Datta, David London, and Shanmuka Shivashankara, Simultaneous Explanation of the $R_{K}$ and $R\left(D^{(*)}\right)$ Puzzles, Phys.Lett., B742 370-374, (2015).

[11] Alejandro Celis, Javier Fuentes-Martin, Martin Jung, and Hugo Serodio, Family non-universal Z' models with protected flavor-changing interactions, , (2015).

[12] Andreas Crivellin, Giancarlo D'Ambrosio, and Julian Heeck, Addressing the LHC flavor anomalies with horizontal gauge symmetries, Phys.Rev., D91(7) 075006, (2015).

[13] Rodrigo Alonso, Benjamín Grinstein, and Jorge Martin Camalich, Lepton universality violation and lepton flavor conservation in B-meson decays, JHEP, 10 184, (2015).

[14] Marat Freytsis, Zoltan Ligeti, and Joshua T. Ruderman, Flavor models for $\bar{B} \rightarrow D^{(*)} \tau \bar{v}$, , (2015).

[15] Andreas Crivellin, Julian Heeck, and Peter Stoffer, A perturbed lepton-specific two-Higgs-doublet model facing experimental hints for physics beyond the Standard Model, Phys. Rev. Lett., 116(8) 081801, (2016).

[16] Martin Bauer and Matthias Neubert, One Leptoquark to Rule Them All: A Minimal Explanation for $R_{D^{(*)}}, R_{K}$ and $(g-2)_{\mu}$, , (2015).

[17] Sofiane M. Boucenna, Alejandro Celis, Javier Fuentes-Martin, Avelino Vicente, and Javier Virto, Phenomenology of an $S U(2) \times S U(2) \times U(1)$ model with lepton-flavour non-universality, , (2016).

[18] S. Aoki et al., Review of lattice results concerning low-energy particle physics, , (2016).

[19] W. Buchmuller and D. Wyler, Effective Lagrangian Analysis of New Interactions and Flavor Conservation, Nucl.Phys., B268 621-653, (1986).

[20] Rodrigo Alonso, Benjamin Grinstein, and Jorge Martin Camalich, $S U(2) \times U(1)$ gauge invariance and the shape of new physics in rare B decays, Phys.Rev.Lett., 113(24) 241802, (2014). 
[21] Christoph Bobeth, Martin Gorbahn, Thomas Hermann, Mikolaj Misiak, Emmanuel Stamou, and Matthias Steinhauser, $B \_s, d->l+l$ - in the Standard Model with Reduced Theoretical Uncertainty, Phys. Rev. Lett., 112 101801, (2014).

[22] Kristof De Bruyn, Robert Fleischer, Robert Knegjens, Patrick Koppenburg, Marcel Merk, et al., Probing New Physics via the $B_{s}^{0} \rightarrow \mu^{+} \mu^{-}$Effective Lifetime, Phys.Rev.Lett., 109 041801, (2012).

[23] Vardan Khachatryan et al., Observation of the rare $B^{0}{ }_{-} s \rightarrow \mu^{+} \mu^{-}$decay from the combined analysis of CMS and LHCb data, Nature, 522 68-72, (2015).

[24] Morad Aaboud et al., Study of the rare decays of $B_{s}^{0}$ and $B^{0}$ into muon pairs from data collected during the LHC Run 1 with the ATLAS detector, , (2016).

[25] Gudrun Hiller and Frank Kruger, More model independent analysis of $b \rightarrow$ s processes, Phys.Rev., D69 074020, (2004).

[26] Marzia Bordone, Gino Isidori, and Andrea Pattori, On the Standard Model predictions for $R_{K}$ and

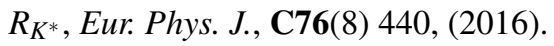

[27] S. Jäger and J. Martin Camalich, On B -\&amp;gt; V l l at small dilepton invariant mass, power corrections, and new physics, JHEP, 1305 043, (2013).

[28] Sebastian JÃd'ger and Jorge Martin Camalich, Reassessing the discovery potential of the $B \rightarrow K^{*} \ell^{+} \ell^{-}$decays in the large-recoil region: SM challenges and BSM opportunities, Phys. Rev., D93(1) 014028, (2016).

[29] Benjamin Grinstein, Martin J. Savage, and Mark B. Wise, $B \rightarrow X_{(s)} e^{+} e^{-}$in the Six Quark Model, Nucl.Phys., B319 271-290, (1989).

[30] M. Beneke, T. Feldmann, and D. Seidel, Systematic approach to exclusive $B \rightarrow V l^{+} l^{-}, V \gamma$ decays, Nucl.Phys., B612 25-58, (2001).

[31] Benjamin Grinstein and Dan Pirjol, Exclusive rare $B \rightarrow K^{*} \ell^{+} \ell^{-}$- decays at low recoil: Controlling the long-distance effects, Phys. Rev., D70 114005, (2004).

[32] M. Beneke and T. Feldmann, Symmetry breaking corrections to heavy to light B meson form-factors at large recoil, Nucl. Phys., B592 3-34, (2001).

[33] J. Charles, A. Le Yaouanc, L. Oliver, O. Pene, and J. C. Raynal, Heavy to light form-factors in the heavy mass to large energy limit of QCD, Phys. Rev., D60 014001, (1999).

[34] Aoife Bharucha, David M. Straub, and Roman Zwicky, $B \rightarrow V \ell^{+} \ell^{-}$in the Standard Model from Light-Cone Sum Rules, , (2015).

[35] A. Khodjamirian, Th. Mannel, and Y.M. Wang, $B \rightarrow K \ell^{+} \ell^{-}$decay at large hadronic recoil, JHEP, 1302 010, (2013).

[36] Sebastien Descotes-Genon, Joaquim Matias, Marc Ramon, and Javier Virto, Implications from clean observables for the binned analysis of $B \rightarrow K^{*} \mu^{+} \mu^{-}$at large recoil, JHEP, 1301 048, (2013).

[37] Sébastien Descotes-Genon, Lars Hofer, Joaquim Matias, and Javier Virto, On the impact of power corrections in the prediction of $B \rightarrow K^{*} \mu^{+} \mu^{-}$observables, JHEP, 1412 125, (2014).

[38] T. Hurth, F. Mahmoudi, and S. Neshatpour, On the anomalies in the latest LHCb data, Nucl. Phys., B909 737-777, (2016).

[39] James Lyon and Roman Zwicky, Resonances gone topsy turvy - the charm of QCD or new physics in $b \rightarrow s \ell^{+} \ell^{-} ?,,(2014)$. 
[40] Marco Ciuchini, Marco Fedele, Enrico Franco, Satoshi Mishima, Ayan Paul, Luca Silvestrini, and Mauro Valli, $B \rightarrow K^{*} \ell^{+} \ell^{-}$decays at large recoil in the Standard Model: a theoretical reappraisal, JHEP, 06 116, (2016).

[41] M. Beylich, G. Buchalla, and T. Feldmann, Theory of $B \rightarrow K^{(*)} \ell^{+} \ell^{-}$decays at high $q^{2}:$ OPE and quark-hadron duality, Eur. Phys. J., C71 1635, (2011).

[42] R Aaij et al., Observation of a resonance in $B^{+} \rightarrow K^{+} \mu^{+} \mu^{-}$decays at low recoil, Phys.Rev.Lett., 111(11) 112003, (2013).

[43] Simon Bra $\tilde{\S}$, Gudrun Hiller, and Ivan Nisandzic, Zooming in on $B \rightarrow K^{*} \ell \ell$ decays at low recoil, , (2016).

[44] Benjamín Grinstein and J. Martin Camalich, Weak Decays of Excited B Mesons, Phys. Rev. Lett., 116(14) 141801, (2016).

[45] C. Glenn Boyd, Benjamin Grinstein, and Richard F. Lebed, Model independent determinations of anti-B $\rightarrow D$ (lepton), $D^{*}$ (lepton) anti-neutrino form-factors, Nucl. Phys., B461 493-511, (1996).

[46] Irinel Caprini, Laurent Lellouch, and Matthias Neubert, Dispersive bounds on the shape of anti-B $\rightarrow$ $D(*)$ lepton anti-neutrino form-factors, Nucl. Phys., B530 153-181, (1998).

[47] Jon A. Bailey et al., Update of $\left|V_{c b}\right|$ from the $\bar{B} \rightarrow D^{*} \ell \bar{v}$ form factor at zero recoil with three-flavor lattice QCD, Phys. Rev., D89(11) 114504, (2014).

[48] Jon A. Bailey et al., $B \rightarrow D \ell v$ form factors at nonzero recoil and $\left|V_{c b}\right|$ from $2+1$-flavor lattice $Q C D$, Phys. Rev., D92(3) 034506, (2015).

[49] Heechang Na, Chris M. Bouchard, G. Peter Lepage, Chris Monahan, and Junko Shigemitsu, $B \rightarrow D \ell v$ form factors at nonzero recoil and extraction of $\left|V_{c b}\right|$, Phys. Rev., D92(5) 054510, (2015).

[50] Rodrigo Alonso, Andrew Kobach, and Jorge Martin Camalich, New physics in the kinematic distributions of $\bar{B} \rightarrow D^{(*)} \tau^{-}\left(\rightarrow \ell^{-} \bar{v}_{\ell} v_{\tau}\right) \bar{v}_{\tau}$, , (2016). 